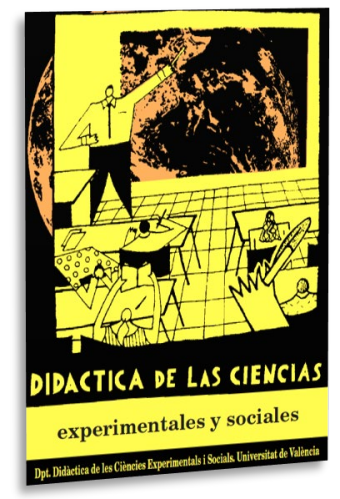

\title{
La enseñanza basada en preguntas: la ley de Ampère y el término de Maxwell
}

\section{Teaching based on questions: Ampère's law and Maxwell's term}

DOI: $10.7203 / D C E S .38 .15427$

\author{
Alberto Sánchez Moreno \\ Centro Interdisciplinario de Investigación y Docencia en Educación Técnica (México) \\ asanchez@ciidet.edu.mx \\ ORCID iD: https://orcid.org/0000-0002-2520-1402 \\ Omar Jaimes Gómez \\ Escuela Nacional de Ciencias Biológicas del IPN (México) \\ omarjaimesg@yahoo.com.mx \\ José Ricardo Aguilera Terrats \\ Centro Interdisciplinario de Investigación y Docencia en Educación Técnica (México) \\ raguilera@ciidet.edu.mx \\ ORCID iD: https://orcid.org/0000-0001-5086-3259
}

\begin{abstract}
RESUMEN: Frecuentemente a los maestros de Física y de Matemáticas se les recomienda una enseñanza activa de estas áreas del conocimiento, que consiste en una interacción continua entre el maestro y el estudiante. Con una metáfora de un interrogatorio adecuado, se pretende mostrar al docente una manera de orientar al estudiante en su aprendizaje, motivándolo a recuperar conocimientos previos o causándole un conflicto cognitivo que lo lleve a reformular su aprendizaje. Esta manera de proceder didácticamente se ha llamado enseñanza basada en preguntas, cuya primera referencia histórica nos remite a la Grecia antigua. En este trabajo se presenta la enseñanza de la ley de Ampère con la generalización de Maxwell mediante la enseñanza basada en preguntas.
\end{abstract}

Palabras Clave: métodos de enseñanza, historia de la ciencia, aprendiendo teoría, enseñando ciencia, electromagnetismo.

ABSTRACT: Often teachers of Physics and Mathematics are encouraged to active teaching of these areas of knowledge, consisting of a continuous interaction between the teacher and the student. With a metaphor of a proper questioning, it is intended to show teachers a way to guide students in their learning, motivating them to recall previous knowledge or causing a cognitive conflict that will lead them to reformulate their learning. This way of proceeding is called teaching based on questions, the first historical reference of which is from ancient Greece. This paper presents the teaching of Ampère's law with Maxwell's generalization through teaching based on questions.

KEYWORDS: teaching methods, history of science, learning theory, science teaching, electromagnetism.

Fecha de recepción: junio de 2019 Fecha de aceptación: febrero de 2020

Los autores agradecen al grupo de Ciencias Básicas del CIIDET sus comentarios y sugerencias a este trabajo. 


\section{INTRODUCCIÓN}

El siglo XIX fue testigo de la síntesis del electromagnetismo. En el libro titulado Un tratado sobre Electricidad y Magnetismo (Maxwell, 1954), escrito por el físico escocés James Clerk Maxwell (1831-1879), se encuentra todo el conocimiento acerca del electromagnetismo desarrollado desde los tiempos de la Grecia antigua (Platón, 1984; Braun, 1992), contenido en un conjunto de ecuaciones que llevan el nombre de "Ecuaciones de Maxwell". Tales ecuaciones involucran uno de los conceptos más importantes de la Física, el concepto de campo electromagnético (Berkson, 1985), junto con otros como los de carga y de corriente eléctrica.

La ley de Ampère es una de estas ecuaciones. En ella se sintetizan los descubrimientos experimentales hechos por el matemático y físico francés André-Marie Ampère (1775-1836) y el físico y químico Danés Hans Christian Oersted (1777-1851) y nos ha permitido tener un entendimiento no solamente conceptual sino aplicado de la naturaleza, por ejemplo, el conocimiento del campo magnético generado por los solenoides (Resnick, Halliday y Krane, 2002; Serway, 1997). Sin embargo, hay otros casos en que es posible producir campo magnético sin necesidad de corriente eléctrica, hecho que llevó a la modificación de la ley de Ampère. Dicha modificación es el famoso término de Maxwell (Tagüeña y Martina, 1988), indispensable para entender el fenómeno de las ondas electromagnéticas.

Por otra parte, la búsqueda de una mejor forma de enseñar Física ha sido motivo de muchas propuestas didácticas (Glynn, 1994; Moreira, 2009). Una de ellas es conocida con el nombre de enseñanza basada en preguntas (Wenning, 2005a), que tiene como objetivo que el maestro guíe al estudiante durante su aprendizaje estableciendo un diálogo con él, donde los conceptos previamente aprendidos, el razonamiento, la reflexión y la discusión estén siempre presentes.

La enseñanza basada en preguntas puede ser usada como una estrategia general a lo largo del plan de estudios de una carrera profesional, lo que sugiere que los docentes debemos plantear la relación de los contenidos con otras asignaturas, tanto las que ya se han cursado como las que se estudian en el mismo ciclo escolar y aquellas que se cursarán en el futuro, actividad que facilita el proceso de transferencia entre un conocimiento obtenido y uno nuevo (Martín del Buey, 1997).

Del mismo modo, esta estrategia bien puede ser implementada como forma de trabajo durante el desarrollo de una materia específica, donde de manera semejante al caso anterior, conviene que el docente plantee preguntas que conduzcan al alumno a relacionar los temas que está estudiando con aquellos que ya se han visto, o bien con aquellos que se trabajarán más adelante. Para ello, conviene apoyarse en organizadores previos, los cuales permiten vincular la información que se ha aprendido con aquella que se habrá de aprender (Díaz-Barriga, 2010).

Esta técnica didáctica puede ser aplicada con fines más limitados debido a la extensión del curso, pero de mayor profundidad, es decir, para la revisión de ciertos objetivos de aprendizaje de un curso, pero que permite al estudiante alcanzar un nivel de razonamiento y reflexión superior, toda vez que se le solicite previamente, revisar los contenidos necesarios para la discusión en clase.

Durante el proceso de interacción del alumno con el maestro, se logra, además de la adquisición del conocimiento propio de la materia, que se den cuenta de sus propias necesidades de aprendizaje y que desarrollen habilidades de análisis y síntesis de información.

Se pretende que, planteándole preguntas adecuadas al alumno, entienda una situación física en particular. Las preguntas también pueden llevar al conflicto cognitivo del estudiante, que es esencial para generar el conocimiento nuevo; aunque es evidente que se requiere tanto de los temas nuevos, así como del repaso de los ya estudiados, para lograr su vinculación y comprensión.

La dinámica de preguntas y respuestas entre docente y estudiante desarrolla el pensamiento crítico indispensable en el estudio de la Física. Intentar responder a las preguntas planteadas, o la discusión mediante el diálogo, tiene la finalidad de que el alumno comprenda y profundice adecuadamente en las respuestas a los problemas que se pretenden resolver o en los temas que se quieren comprender, pues durante la interacción intervienen aspectos de orden filosófico, 
sociológico, psicológico, histórico, práctico y conceptual. Todo lo anterior con un enfoque integral. La estructura y el proceso de la enseñanza basada en preguntas están siempre abiertos, lo que motiva un aprendizaje consciente y una experiencia colaborativa de aprendizaje docente-estudiante.

Este artículo presenta a manera de metáfora, dos ejemplos de enseñanza basada en preguntas, considerando dos temas fundamentales del electromagnetismo: la ley de Ampère y su generalización con el término de Maxwell. Ambos son temas de interés para los docentes de las diferentes escuelas de nivel superior que imparten carreras de Ingeniería.

El trabajo está organizado de la siguiente manera: en la sección 2, se presenta una breve revisión del enfoque didáctico conocido con el nombre de enseñanza basada en preguntas. En la sección 3, se hace una reseña histórica acerca del término de Maxwell. En las secciones 4 y 5, se dan ejemplos de la enseñanza basada en preguntas para el caso de la Ley de Ampère y el término de Maxwell, ambos a manera de metáfora en la conversación entre el docente y un estudiante, donde no se espera que se dé esta situación ideal, sino que se pretende mostrar el tipo de razonamientos que se requieren que los estudiantes logren realizar a partir de la reflexión y la documentación. Finalmente, la sección 6 se dedica a las conclusiones.

\section{ENSEÑANZA UTILIZANDO PREGUNTAS}

Tratar de enseñar algún concepto o tema mediante un interrogatorio dirigido no es un método didáctico novedoso. En la Grecia antigua ya había sido propuesto por Platón en su diálogo Menón o de la virtud (Platón, 1984), en donde, en boca de Sócrates, utilizando preguntas dirige el razonamiento de un esclavo para que resuelva un problema. Esta forma de enseñar, que consiste esencialmente en descomponer un problema en partes más sencillas y fáciles de entender, por medio de preguntas que induzcan al estudiante a ver con claridad cuál es la respuesta al problema, también se encuentra presente en el Discurso del Método y sus Reglas para la Dirección del Espíritu de Descartes (2003), reglas $\mathrm{V}$ y IX.

Si consideramos que esta manera de enseñar es un intento para que el estudiante, con sus propios medios, logre razonar y descubrir la solución a los problemas, también podríamos citar a Leibniz como precursor de esta idea, ya que él afirmaba: "Nada es más importante que descubrir las fuentes de la invención que, en mi opinión, son más valiosas que la invención misma" (Polya, 1989, p. 120)

Recientemente, este método de enseñanza ha sido motivo de diversas investigaciones (Costenson y Lawson, 1986; Wenning, 2005a; Wenning, 2005b; Wenning, 2010), a partir de las cuales se ha desarrollado toda una clasificación conocida como "Espectro científico de Preguntas" (Wenning, 2010), que se muestra en la imagen 1.

IMAGEN 1. Espectro científico de preguntas.

\begin{tabular}{|l|l|l|l|l|l|}
\hline $\begin{array}{l}\text { Aprendizaje por } \\
\text { descubrimiento }\end{array}$ & $\begin{array}{l}\text { Demostraciones } \\
\text { interactivas }\end{array}$ & $\begin{array}{l}\text { Clase de } \\
\text { preguntas }\end{array}$ & $\begin{array}{l}\text { Laboratorio } \\
\text { de preguntas }\end{array}$ & $\begin{array}{l}\text { Aplicaciones } \\
\text { al mundo real }\end{array}$ & $\begin{array}{l}\text { Preguntas } \\
\text { hipotéticas }\end{array}$ \\
\hline
\end{tabular}

Fuente: Elaboración propia

Cada uno de estos métodos de enseñanza se basa en la dirección del maestro mediante preguntas orientadas, y tienen como propósito que en el estudiante se desarrolle el conocimiento y el entendimiento de ideas científicas, así como la comprensión de la manera en que la ciencia estudia la naturaleza (National Research Council, 1996).

En el caso de una clase con base en preguntas, la intención didáctica consiste en que el maestro poco a poco guíe y conduzca al estudiante, mediante el interrogatorio, a formular sus propias conclusiones, a identificar las variables que está utilizando y a definir el sistema que están estudiando. En este método se le pide al alumno que demuestre que entiende el problema a resolver; después, el 
maestro explica y comenta la naturaleza de sus preguntas. El propósito didáctico es que el estudiante identifique principios y/o relaciones científicas. Entre las habilidades que se desea que el alumno desarrolle están el uso e interpretación de relaciones matemáticas, así como diseñar y conducir investigaciones científicas (Wenning, 2005a; Wenning, 2005b).

Otro aspecto importante a considerar, para el éxito de esta manera de instruir, es asegurarse (mediante un interrogatorio adecuado) de que los alumnos poseen los antecedentes necesarios para el tema que se pretende enseñar. También es recomendable que el maestro anticipe a la clase las ideas principales, el plan general, las grandes líneas del desarrollo histórico del tema que se propone tratar en la lección, con el fin de que el estudiante adquiera un conocimiento dinámico del tema que le permita reconstruirlo, en caso de ser necesario, sin ayuda del maestro y del texto.

En este contexto, se han llevado a cabo numerosas investigaciones que proponen el uso de la historia como herramienta didáctica para el aprendizaje de la Física (Solbes, Pomer y Tarín, 1997; Arons ,1970; Holton y Brush, 1976). Dado que la enseñanza del campo electromagnético ha sido una tarea particularmente compleja (Martín y Solbes, 2001; Solbes y Martín, 1991), el conocimiento histórico del desarrollo de los conceptos de campo eléctrico y campo magnético, así como de las leyes que cumplen, como es el caso que ocupa a este artículo, ayuda al proceso de enseñanza-aprendizaje del alumno. En la sección 3, presentamos una breve revisión de los antecedentes históricos relacionados con la corriente de desplazamiento y las ecuaciones de Maxwell, considerando la conveniencia de que este conocimiento histórico sirva como antecedente a la propuesta didáctica aquí sugerida y ejemplificada en las secciones 4 y 5 .

\section{ANTECEDENTES}

Después de que Maxwell dedujo y publicó las ecuaciones que describen los campos eléctrico y magnético estáticos, se dio a la tarea de investigar para descubrir qué sucedía en el caso de las cantidades involucradas en sus ecuaciones que tuvieran dependencia temporal. Para resolver el problema, Maxwell postuló un modelo mecánico para el campo electromagnético, el cual debía estar de acuerdo con las siguientes observaciones experimentales (Maxwell, 1865):

- La fuerza entre cargas eléctricas en reposo.

- La fuerza entre polos magnéticos.

- El campo eléctrico que crea una corriente circular.

- La corriente eléctrica que crea un campo eléctrico cambiante en una espira, el cual tenía que describir.

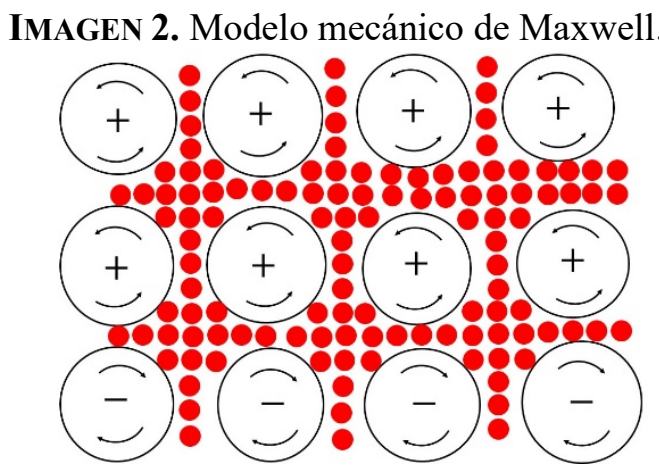

Fuente: Elaboración propia

El modelo mecánico de Maxwell consistía en postular la existencia de cierto mecanismo que servía como base al campo electromagnético. Dicha base consistía en diminutas esferas que llenaban todo el espacio, cuya densidad era muy baja, y que eran capaces de rotar (ver imagen 2). Cuando una de tales esferas gira, la fuerza centrípeta le cambia su forma, como le pasa a la Tierra que se achata 
en los polos y se ensancha en el ecuador. Esto provocará un empuje a la esfera contigua, y si ocurre en todas las esferas, el efecto conjunto será una presión efectiva en la dirección perpendicular al eje de rotación. En la dirección del eje de rotación sucede exactamente lo contrario, ya que las esferas tienden a contraerse (ver imagen 3). Por tanto, si todas las esferas están alineadas, el eje de rotación y las direcciones perpendiculares a éste se comportan como las líneas de fuerza de Faraday, una fuerza de atracción a lo largo del eje de rotación y una fuerza de repulsión en las direcciones perpendiculares a la misma.

IMAGEN 3. Esquema que muestra la atracción y repulsión de acuerdo con el modelo mecánico de Maxwell.

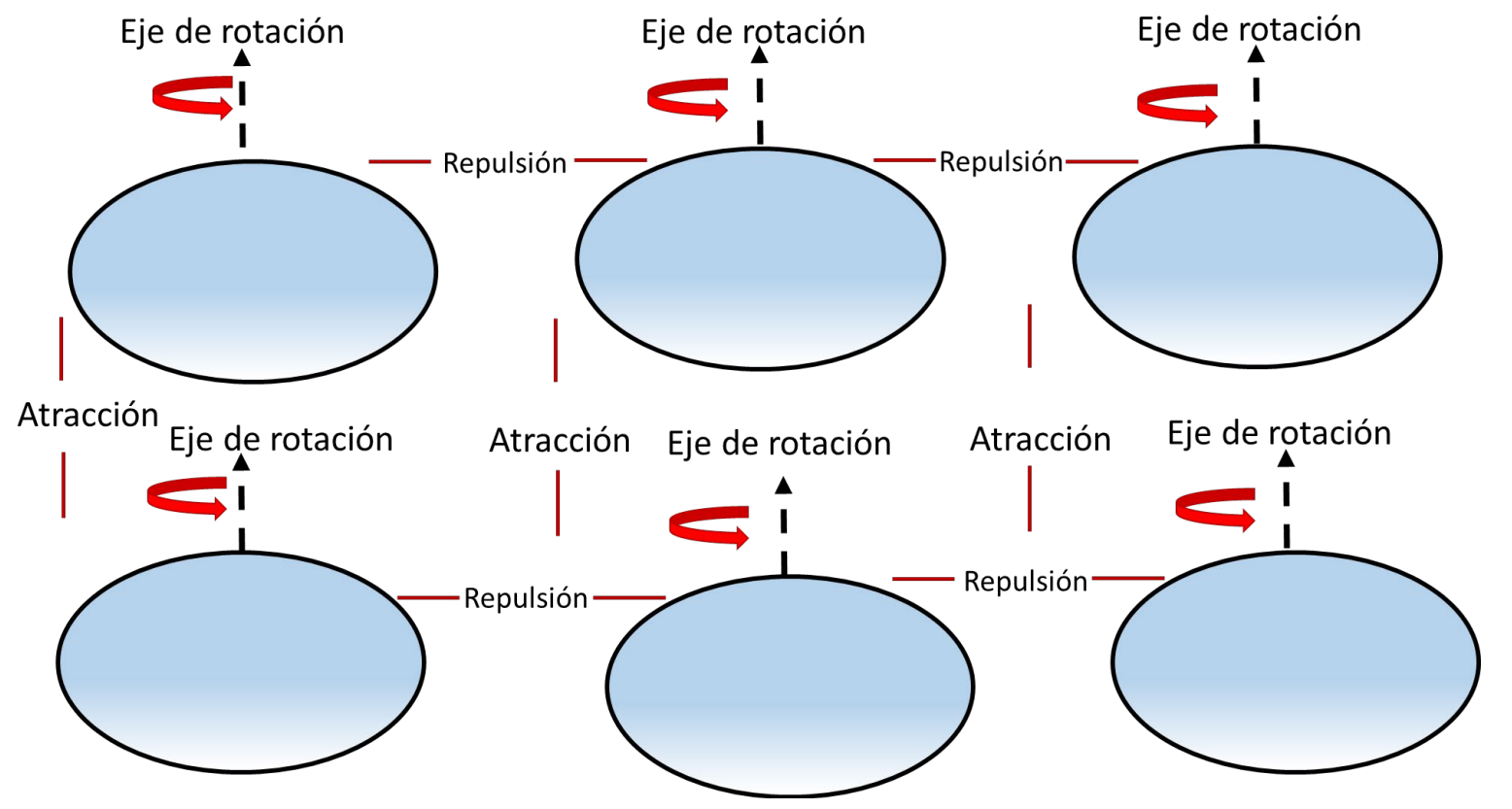

Fuente: Elaboración propia

La posibilidad de giro de las esferas en sentido horario o antihorario permitía explicar el campo negativo y el positivo. Por tanto, los ejes de rotación de las esferas definían la dirección del campo magnético en cualquier punto del espacio; y su densidad y velocidad de rotación, la intensidad del mismo. Para evitar que las esferas frenaran su rotación por fricción, Maxwell postuló que entre ellas existían otras esferas más pequeñas, que actuaban como cojinetes (como los baleros de las bicicletas), y supuso que eran partículas eléctricas, y que su desplazamiento provoca que las otras esferas comiencen a rotar.

Agregando elasticidad a su modelo, Maxwell fue capaz de explicar todos los fenómenos electromagnéticos, además de concebir la idea de las ondas electromagnéticas. Este modelo también permitió predecir que aparecían pequeñas corrientes eléctricas en el espacio vacío, cuando el campo eléctrico variaba con el tiempo. A esta corriente la llamó "corriente de desplazamiento". Tal predicción fue una de las aportaciones más importantes de Maxwell al electromagnetismo. Sin embargo, a pesar del éxito del modelo, debido a que estaba sustentado en la idea del éter -esa sustancia invisible y con propiedades exóticas que se consideraba llenaba todo el espacio-, no era del agrado de todo el mundo. El propio Maxwell consideraba que su modelo era muy insatisfactorio, que se trataba de una hipótesis provisional para explicar los fenómenos electromagnéticos y que debía buscar en las matemáticas y los principios de la dinámica otra explicación.

Utilizando la teoría del matemático francés Joseph-Louis Lagrange (1736-1813) y el concepto de conservación de la energía, Maxwell fue capaz de derivar, sin necesidad del éter, un conjunto de ecuaciones que resolvían el problema de los campos variables. Este trabajo fue presentado en una reunión de la Royal Society en diciembre de 1864 (Maxwell, 1865). En palabras de Maxwell: 
La teoría que propongo podría llamarse una teoría del campo electromagnético, porque tiene que ver con el espacio situado en la vecindad de los cuerpos eléctricos y magnéticos, y puede llamarse una teoría dinámica porque supone que en el espacio hay materia en movimiento, que es la que produce los fenómenos electromagnéticos observados. (Maxwell, 1865, p. 460)

Una de dichas ecuaciones era la ley de Ampère, que establece, como ya lo mencionamos, que una corriente eléctrica estacionaria genera un campo magnético estático, y que Maxwell corrigió al introducir la corriente de desplazamiento.

\section{LA LEY DE AMPÈRE}

En 1823 el astrónomo francés Felix Savart (1797-1841) y el profesor de Matemáticas y Física Jeand-Firmin De Montferrand (1795-1844) lograron una deducción de la ley de Biot-Savart, a partir de los trabajos de Ampère (Pérez y Varela, 2003; Reitz, Milford y Christy, 1996). Con esto se daba certeza y veracidad a las investigaciones experimentales y matemáticas realizadas por Ampère. Mediante esta ley se había logrado expresar la relación existente entre la corriente eléctrica estacionaria $I$ y la magnitud del campo magnético $B$, que dicha corriente crea a una cierta distancia $r$, de la misma, cuya expresión matemática es la siguiente:

$$
B=\frac{\mu I}{2 \pi r}
$$

donde $\mu$ es una cantidad constante conocida como la permeabilidad magnética. Entonces, Ampère, a partir de esa expresión, dedujo una relación general entre las dos magnitudes. Sea cual sea la forma del conductor por el que circula la corriente de intensidad constante $I$, ésta es

$$
\oint \vec{B} \cdot d \vec{l}=\mu \sum_{i} I_{i}
$$

La ecuación nos dice que la integral de trayectoria cerrada (circulación) del campo vectorial $\vec{B}$, es igual al producto de la permeabilidad magnética $\mu$, por la intensidad de corriente eléctrica resultante creadora de dicho campo (suma algebraica de las intensidades de corriente que atraviesan la superficie limitada por esa línea cerrada).

\subsection{La clase basada en preguntas}

A continuación, se muestra una metáfora de una clase basada en preguntas, acerca de la Ley de Ampère, cuyo diseño representa los elementos que debe considerar el docente para utilizar esta técnica. Cabe mencionar que no se trata de una dinámica de clase cotidiana, sino que se busca enfatizar en los siguientes aspectos:

1. El profesor ya conoce los saberes previos de los estudiantes, lo que puede hacerse mediante una prueba de diagnóstico.

2. Se cuenta con un laboratorio para realizar experimentos de electromagnetismo.

3. Las preguntas son de tres tipos: para recordar (r), detonar el conflicto cognitivo (cc) y propiciar la transferencia $(\mathrm{t})$.

4. Las respuestas del estudiante no reflejan al alumno promedio, sino el tipo de razonamiento que se busca propiciar en el grupo.

Con respecto al último aspecto, se debe hacer notar que el modelo de estudiante utilizado para llevar a cabo la presente propuesta no es el que regularmente se encuentra en una clase real, sino más bien uno totalmente idealizado que no tiene las carencias que posiblemente pueda tener un alumno verdadero, a quien sí se le tendrá que solicitar realice lectura previa y posterior a la clase.

Con base en las consideraciones anteriores, el ejemplo de la clase comenzaría de la siguiente manera: 
Maestro: Antes de iniciar la lección de hoy, conversemos acerca del concepto de campo, uno de los conceptos fundamentales de la Física. Para precisar y facilitar la discusión, pensemos en el campo magnético. ¿Cómo imagina usted el campo magnético?

Alumno: Considero que ésa es una pregunta muy complicada porque no me puedo imaginar algo que en esencia no existe, ya que no lo puedo tocar, ver, oír, oler o distinguir su sabor; es decir, detectarlo por medio de mis sentidos. Honestamente, creo que mi respuesta sería, no sé.

Maestro: Me parece que su apreciación es correcta hasta cierto punto, sin embargo, discutamos un poco más con respecto a detectar el campo. Veamos, ¿cuándo tuvo su primer conocimiento acerca del campo magnético?

Alumno: Fue en el laboratorio de Física. Al experimentar con imanes, observamos que se atraían y se repelían. La explicación que nos dio el profesor es que esto sucedía por mediación del campo magnético que producen los imanes.

Maestro: ¿Qué pasaría si solamente tuviera un imán? ¿De acuerdo con la experiencia mencionada, podría conocer el campo magnético de dicho imán?

Alumno: Nuevamente me pone en dificultades, pero recuerdo de mi curso de secundaria que colocando una hoja de papel encima del imán y esparciendo en ella limadura de fierro se dibujaba el campo magnético.

Maestro: Y si tampoco tuviera dicha limadura de fierro, ¿podría conocer el campo magnético? Alumno: Me parece que la respuesta es ¡No!

Maestro: Esa es la respuesta correcta, y es porque se necesita que algo detecte el campo magnético, en otras palabras, algo que pruebe su existencia. Es en este sentido que decimos que el campo magnético no lo podemos percibir por medio de nuestros sentidos, pero sí lo podemos detectar indirectamente. Claro está que lo que prueba la existencia del campo magnético tiene una característica especial, la propiedad de campo magnético. Pero sigamos adelante, ¿de qué otra manera se puede producir campo magnético además de los imanes?

Alumno: He leído que en el siglo XIX un físico danés de nombre Hans Christian Oersted, realizó experimentos con brújulas y alambres con corriente y observó que las brújulas eran deflectadas cuando circulaba corriente en los alambres. Si consideramos que las brújulas son imanes, y de la discusión anterior sabemos que los imanes sólo interaccionan con imanes a través del campo magnético, puedo deducir que el alambre actúa como un imán generando un campo magnético.

Maestro: Está usted en lo cierto. Uno de los grandes descubrimientos en investigación electromagnética es que la corriente eléctrica produce campo magnético. Esto fue descubierto y demostrado, como ya lo ha indicado antes, por Oersted y Ampère en el siglo XIX. Ahora, consideremos un alambre recto largo de sección transversal constante y coloquemos imanes de barra a su alrededor a varias distancias diferentes $r_{i}$, medidas desde el alambre. ¿Qué sucederá con este sistema?

Alumno: Los imanes se alinearán en dirección norte sur geográfica.

Maestro: ¿Cómo lo sabe?

Alumno: Los imanes no son más que brújulas y las brújulas tienen esa orientación. Además, lo he experimentado.

Maestro: Excelente. Consideremos ahora que en el alambre hacemos circular una corriente $I$, ¿qué sucederá ahora con los imanes?

Alumno (después de hacer el experimento): Los imanes se disponen tangentes a círculos concéntricos, al alambre, de radio $r_{i}$ (ver imagen 4).

Maestro: ¿Qué modelo matemático describirá este fenómeno físico? 
Alumno: De mi conocimiento de cálculo vectorial, puedo decir que el conjunto de imanes será un conjunto de flechas. Entonces, matemáticamente podemos decir que tenemos un conjunto de vectores, un campo vectorial.

IMAGEN 4. Esquema de imanes en el campo magnético generado por la corriente $\boldsymbol{I}$ saliendo de la página.

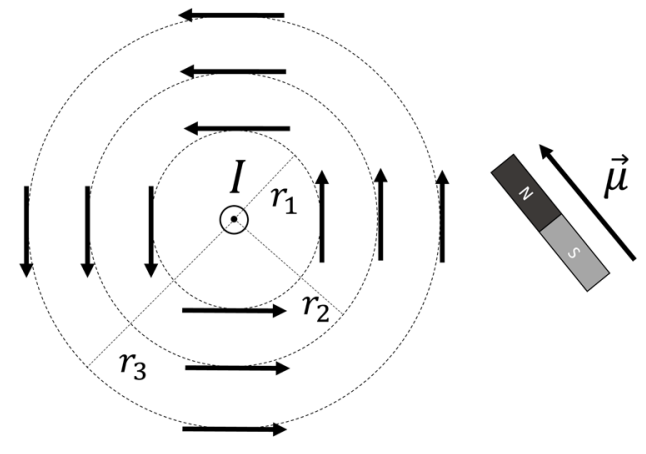

Fuente: Elaboración propia

Maestro: Muy bien. Denotemos a estos vectores con el símbolo $\vec{\mu}$. Si consideramos que la dirección de $\vec{\mu}$ es la dirección del campo magnético producido por la corriente en el alambre, ¿cómo podemos representar matemáticamente dicho campo?

Alumno: Mediante un vector. Tendremos un campo vectorial magnético.

Maestro: $\mathrm{Su}$ respuesta es correcta. Denotemos este campo como $\vec{B}$. Ahora, en estas circunstancias, ¿qué debemos hacer para girar los imanes un ángulo $\theta$ con respecto a su posición de equilibrio, que es aquella cuando no había corriente en el alambre?

Alumno: Me parece que necesito mis conocimientos de mecánica. Según recuerdo, debemos ejercer una torca lo suficientemente grande como para contrarrestar la torca restauradora que actúa sobre los imanes.

Maestro: ¿Recuerda esta expresión?

Alumno: Me parece que sí, es:

$$
\tau=\mu B \sin \theta
$$

Maestro: ¿Está usted seguro? Me parece que ha olvidado algo fundamental, ¿qué clase de cantidad es la torca?

Alumno: Es cierto. Ahora recuerdo. La torca es una cantidad vectorial, entonces la ecuación que he mencionado representa la magnitud de la torca.

Maestro: Efectivamente, que nunca se le olvide, y si esto es así ¿cuál será la expresión correcta para la torca?

Alumno: Considerando que tanto el imán como el campo magnético están representados por vectores, y puesto que en la magnitud aparece la función seno, estas cantidades deben estar relacionadas con un producto cruz:

$$
\vec{\tau}=\vec{\mu} \times \vec{B}
$$


IMAGEN 5. Imán haciendo un ángulo $\theta$ con respecto a su posición de equilibrio.

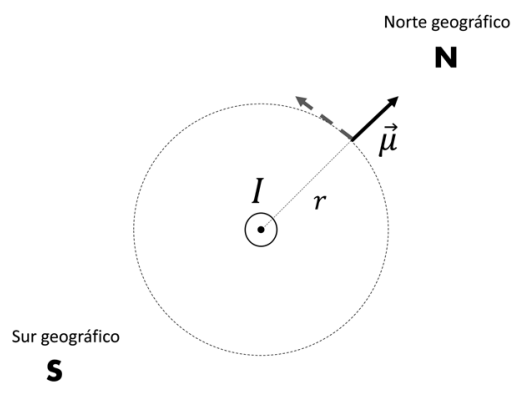

Fuente: Elaboración propia

Maestro: Correcto. Continuemos. Aunque no sabemos el valor de $\mu$, sabemos que es constante e independiente de su posición y orientación. ¿Cómo podríamos conocer el valor de $B$ a partir de la expresión (3)?

Alumno: Este es un problema experimental. Si se me permite especular, propongo aplicar una fuerza a los imanes para regresarlos a su posición de equilibrio, medir el valor de esa fuerza con algún instrumento y después calcular la torca considerando brazo de momento a partir del punto de giro del imán, así tendremos una serie de datos que podríamos graficar para obtener un valor relativo del campo magnético (ver imagen $5)$.

Maestro: Magnífico. Este razonamiento suyo nos llevaría a tener la representación gráfica (ver imagen 6) entre la torca y el seno del ángulo que es una relación lineal, cuya pendiente de la gráfica nos permitiría calcular el campo magnético (un múltiplo de él por medio de una constante de proporcionalidad). ¿Cómo relacionaría el campo magnético, así encontrado, con la corriente y la distancia al alambre?

Imagen 6. Gráfica de $\tau v \boldsymbol{s} \sin \theta$.

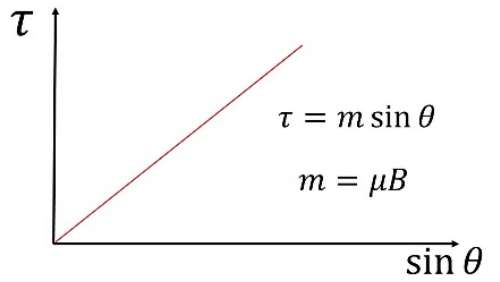

Fuente: Elaboración propia

Alumno: Llevaría a cabo el mismo procedimiento para diferentes corrientes y distancias al alambre para encontrar la manera en que se relacionan estas cantidades.

Maestro: De acuerdo. El resultado que obtendría sería el mismo que encontró Ampère: el campo magnético es directamente proporcional a la intensidad de corriente e inversamente proporcional a la distancia al alambre, esto es

$$
B \propto \frac{I}{r} \text {. }
$$

Para considerar la igualdad, es necesaria una constante que tiene el valor $\frac{\mu_{0}}{2 \pi}$, tomando en cuenta el vacío, con lo cual la forma final buscada es

que se puede poner en la forma

$$
B=\frac{\mu_{0} I}{2 \pi r}
$$

$$
B(2 \pi r)=\mu_{0} I \text {. }
$$


Detengámonos aquí para hacer un poco de cálculo vectorial. Considere nuevamente la figura 4. ¿Cuál es el valor de la integral de línea (circulación) del campo magnético $\vec{B}$ a través de la trayectoria circular concéntrica al alambre (el perímetro del círculo al cual son tangentes los imanes del experimento)?

Alumno: De mi curso de cálculo vectorial, sé que esto lo podemos calcular mediante la expresión

$$
C=\oint_{l} \vec{B} \cdot d \vec{l}
$$

con $d l$, un elemento diferencial del perímetro del círculo de radio $r$. Calculando, tenemos

$$
C=\oint_{l} B d l \cos 0^{0}=B \oint_{l} d l=B(2 \pi r)
$$

Maestro: Bien calculado. Con base en las ecuaciones 7 y 9, ¿qué puede concluir de esto?

Alumno: Si comparamos la ecuación (9) con la ecuación (7), podemos concluir que

$$
\oint_{l} \vec{B} \cdot d \vec{l}=\mu_{0} I
$$

Maestro: Su conclusión es correcta. La ecuación (10) recibe el nombre de Ley de Ampère en forma integral, y un gran número de experimentos diferentes sugieren que esta ecuación es válida en general con cualquier configuración de campos magnéticos, con cualquier conjunto de corrientes y en cualquier trayectoria de integración. La ley de Ampère es análoga a la ley de Gauss, en el sentido de que, conociendo la fuente, en este caso la corriente, se puede calcular el campo magnético. De la misma manera que en la ley de Gauss, si conocemos la carga podemos calcular el campo eléctrico. Al igual que en la ley de Gauss, donde la superficie cerrada debe contener la carga, en caso contrario la integral de superficie es cero. En el caso de la ley de Ampère, la trayectoria de integración debe delimitar el área que cruza la corriente. De no ser así, el resultado de la circulación también será cero. Siguiendo con cálculo vectorial, ¿recuerda usted el teorema de Stokes?

Alumno: Me parece que sí, pero no estoy seguro.

Maestro: No se preocupe, este teorema nos dice cómo cambiar una integral de línea por una integral de superficie, y para mantener la igualdad de expresiones hay que cambiar el integrando por el rotacional de campo vectorial. ¿Podría aplicar esta afirmación a la Ley de Ampère?

Alumno: Me parece que sí, esto es,

$$
\oint_{l} \vec{B} \cdot d \vec{l}=\int_{S}(\nabla \times \vec{B}) \cdot d \vec{a} .
$$

Maestro: Muy bien. Ahora, ¿recuerda cuál es la definición de la corriente eléctrica en términos del vector densidad de corriente eléctrica?

Alumno: Cuando vimos el tema corriente eléctrica, hablamos de dicho vector. Se denota como $\vec{J}$ y físicamente representa la cantidad de corriente por unidad de área en un conductor, y se define mediante la relación general

$$
I=\int_{S} \vec{J} \cdot d \vec{a}
$$

Maestro: Ha comprendido correctamente. Si utilizamos las ecuaciones (11) y (12) en la ley de Ampère obtenemos

$$
\int_{S}(\nabla \times \vec{B}) \cdot d \vec{a}=\mu_{0} \int_{S} \vec{J} \cdot d \vec{a},
$$

de donde, factorizando, obtenemos la expresión 


$$
\int_{S}\left(\nabla \times \vec{B}-\mu_{0} \vec{J}\right) \cdot d \vec{a}=0
$$

Para que se cumpla esta ecuación, tiene que suceder que

$$
\nabla \times \vec{B}=\mu_{0} \vec{J}
$$

La ecuación (15) recibe el nombre de ley de Ampère en forma diferencial. Me parece que hasta aquí podemos concluir la lección.

\section{El término de MAXWell}

Desafortunadamente, a pesar de su utilidad, la ley de Ampère no es una ley fundamental, ya que no describe todos los fenómenos magnéticos; para que lo sea es necesario modificarla. Dicha tarea la realizó, como ya hemos mencionado en la sección I, J.C. Maxwell, quien modificó la ley de Ampère al incluir un término que tenía que ver con la variación del campo eléctrico con el tiempo, relacionado con la corriente de desplazamiento. El término de Maxwell es esencial en dos sentidos: el primero, porque nos permite entender que es posible generar campo magnético en regiones donde no hay corriente eléctrica (el problema del capacitor que discutiremos con detalle a continuación); el segundo, porque permite comprender una ley fundamental de conservación electromagnética, la conservación de la carga.

\subsection{La clase basada en preguntas}

Ahora, presentamos una metáfora que alude a los elementos a considerar en una clase de los primeros cursos de las carreras de ciencias e ingeniería basada en la estrategia de enseñanza a través de preguntas. Es importante basarse en las mismas consideraciones que se mencionaron en la sección 4.1.

Maestro: Considere el siguiente experimento: un capacitor de placas paralelas está siendo cargado por medio de un alambre de corriente como lo muestra la imagen 7. ¿Podría indicar en qué lugares de este sistema físico hay campo magnético?

IMAGEN 7. Capacitor de placas paralelas cargándose por medio de la corriente.

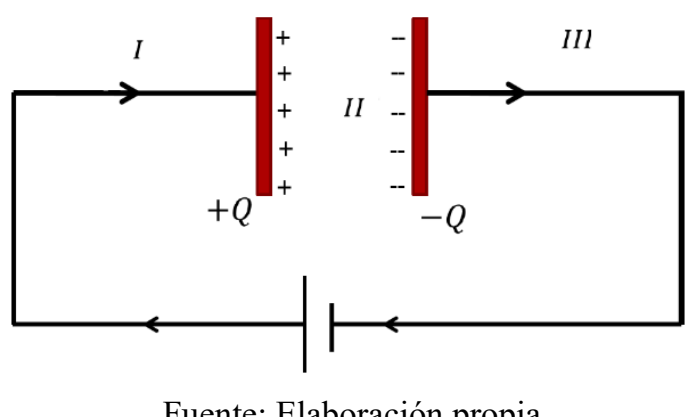

Fuente: Elaboración propia

Alumno: La pregunta me resulta sencilla. De acuerdo con las lecciones que hemos estudiado, el campo magnético es generado por imanes o por corrientes eléctricas. En este caso, sólo existen corrientes eléctricas en las zonas marcadas con los números romanos I y III; por tanto, allí tendremos campo magnético. En la zona II, definitivamente no habría campo magnético porque es una región vacía.

Maestro: Me parece adecuada su respuesta. ¿Cómo demostraría su aseveración?

Alumno: Utilizando la ley de Ampère, es decir, calculando las integrales de trayectoria cerrada de la siguiente manera (ver imagen 8 ): 
IMAGEN 8. Cálculos de campo magnético realizados por el alumno.

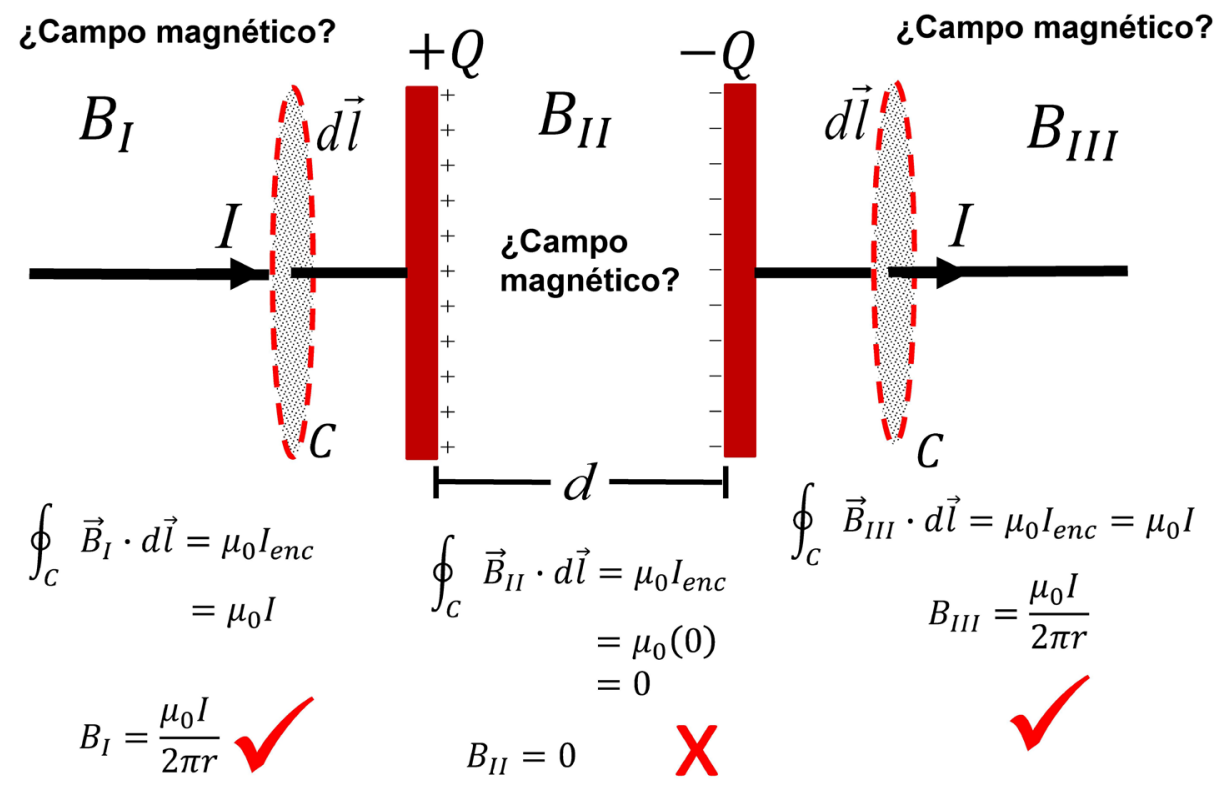

Fuente: Elaboración propia

Maestro: Sus cálculos me parecen impecables. Ahora, ¿cómo lo mostraría experimentalmente?

Alumno: No parece una tarea complicada. Tendría que probar el campo en cada una de las zonas. Una brújula me parece un objeto de prueba adecuado. Veamos (ver imagen 9).

Maestro: Que su brújula haya marcado la existencia de un campo magnético en la región II me sorprende. Es un resultado que intuitivamente no se espera. ¿Qué pasó? ¿Cómo podemos explicar este problema?

Alumno: Dado que la evidencia experimental es contundente, no queda más que pensar que se trata de un problema de cálculo. Aunque me cueste admitirlo, es un problema de la Ley de Ampère. No sé cómo resolverlo.

IMAGEN 9. Esquema donde se ilustra que entre las placas del capacitor hay campo magnético.

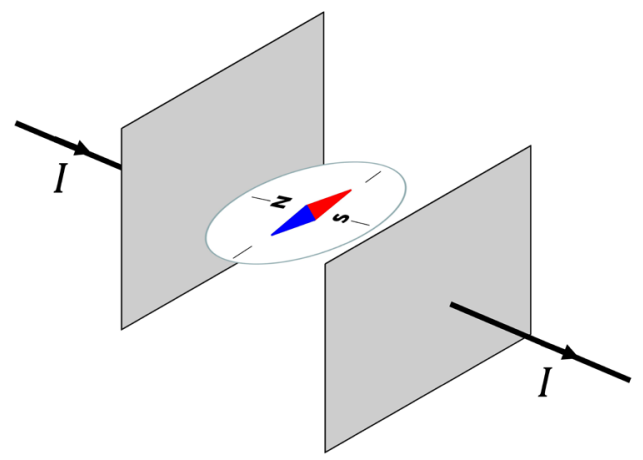

Fuente: Elaboración propia

Maestro: No se preocupe, porque no es un problema fácil de resolver. Fue necesaria una mente brillante como la del físico escocés J.C. Maxwell para lograrlo. Él lo solucionó considerando la validez de la ley de Gauss y definiendo el concepto de corriente de desplazamiento. Para entenderlo, utilicemos la superficie $S$ que encierra una de las placas del capacitor como lo muestra la imagen 10. 
IMAGEN 10. La superficie $\boldsymbol{S}$ está constituida por las superficies $\boldsymbol{S}^{\prime}$ y $\boldsymbol{S}^{\prime \prime}$.

El campo eléctrico $\overrightarrow{\boldsymbol{E}}$ fluye a través de la superficie $\boldsymbol{S}$.

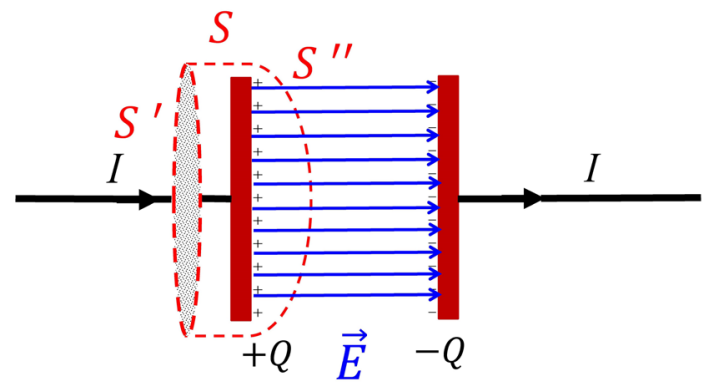

Fuente: Elaboración propia.

Dicha superficie está formada por el área $S^{\prime}$, cuyo perímetro es el del círculo de radio $r$ y la superficie $S^{\prime \prime}$. En la parte superior (círculo de radio $r$ ), hay corriente pero no flujo eléctrico. A través del resto de la superficie no hay corriente, sino un flujo eléctrico que varía con el tiempo porque el capacitor se está cargando con la corriente y, por tanto, la carga en los electrodos es función del tiempo. ¿Cuál es el valor de la carga en este capacitor?

Alumno: De acuerdo con la definición de capacitancia, la carga $Q$ en el capacitor está dada por la relación

$$
Q=C V_{a b},
$$

donde $C$ representa a la capacitancia y $V_{a b}$ la diferencia de potencial entre los electrodos del capacitor.

Maestro: De acuerdo. Entonces, si utilizamos los valores para $C$ y $V_{a b}$, calculados en clases pasadas, en la ecuación (16) obtenemos

$$
Q=\left[\frac{\epsilon_{0} A}{d}\right][E d]=\epsilon_{0} E A,
$$

donde $E$ representa al campo eléctrico, $A$ el área de las placas del capacitor y $d$ la separación entre ellas. Ahora, volvamos al cálculo vectorial. ¿Puede usted decir qué representa el producto $E A$ ?

Alumno: De acuerdo con el cálculo vectorial, $E A$ representa el flujo del campo vectorial a través de la superficie $A$, que podemos representarlo como

$$
\Phi_{\vec{E}}=E A \text {. }
$$

Maestro: Excelente. Si derivamos la ec. (17) con respecto al tiempo, obtenemos

$$
\frac{d Q}{d t}=\epsilon_{0} \frac{d \Phi_{\vec{E}}}{d t}
$$

¿Qué puede concluir de esta ecuación?

Alumno: No puedo dejar de manifestar mi sorpresa. La cantidad $\frac{d Q}{d t}$ define una corriente eléctrica.

Maestro: Efectivamente. Se conoce con el nombre de corriente de desplazamiento y se denota como $I_{D}$ ¿ ¿De qué manera incluiría este término en la ley de Ampère?

Alumno: Dado que la ley de Ampère es una expresión que relaciona el campo magnético con la fuente que lo produce y ésta se encuentra en el lado derecho de la igualdad, yo colocaría la nueva corriente del mismo lado que la otra, ya que allí deben estar todas las fuentes de campo magnético.

Maestro: Su razonamiento me parece correcto, por lo que podemos modificar la ley de Ampère quedando como se muestra a continuación: 


$$
\oint_{l} \vec{B} \cdot d \vec{l}=\mu_{0}\left(I+I_{D}\right)
$$

Utilizando la expresión (19), esta última ecuación se puede poner en la forma

$$
\oint_{l} \vec{B} \cdot d \vec{l}=\mu_{0} I+\mu_{0} \epsilon_{0} \frac{d \Phi_{\vec{E}}}{d t}
$$

que es la ecuación que representa a la ley de Ampère con el término de Maxwell o la ley Ampère-Maxwell en su forma integral. ¿Podría mostrar cómo el nuevo término resuelve el problema del capacitor?

Alumno: Con gusto, maestro. Si aplicamos la nueva relación a la región entre las placas del capacitor, tendremos

$$
\oint_{l} \vec{B} \cdot d \vec{l}=\mu_{0} \epsilon_{0} \frac{d \Phi_{\vec{E}}}{d t},
$$

ya que entre las placas no hay corriente eléctrica (ver imagen 11). Ahora, al utilizar la ecuación (19) en la ecuación (22), se tiene que

$$
\oint_{l} \vec{B} \cdot d \vec{l}=\mu_{0} I_{D}
$$

IMAGEN 11. Región donde se aplica la ley de Ampère-Maxwell. Entre las placas existe un flujo de campo eléctrico que varía con el tiempo.

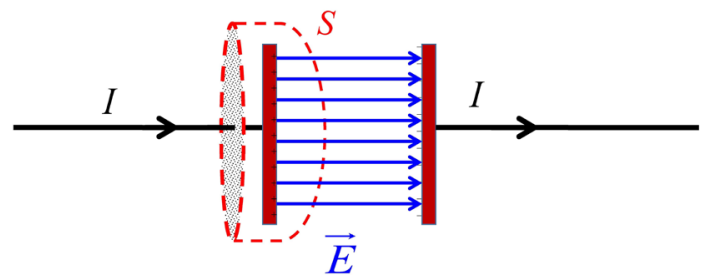

Fuente: Elaboración propia

Lo que nos dará el mismo resultado de campo magnético que obtuvimos cuando hicimos el cálculo utilizando los alambres con corriente eléctrica, ya que $I_{D}=\frac{d Q}{d t}$.

Maestro: Muy bien. ¿Qué debemos hacer ahora para obtener la ley de Ampère-Maxwell en su forma diferencial?

Alumno: Este es un caso más para el uso del Teorema de Stokes. Si lo aplicamos a la relación (21), y consideramos la definición de la corriente $I$ y el flujo $\Phi_{\vec{E}}$ en términos de los vectores densidad de corriente y campo eléctrico, obtenemos

$$
\int_{S}(\nabla \times \vec{B}) \cdot d \vec{a}=\mu_{0} \int_{S} \vec{J} \cdot d \vec{a}+\mu_{0} \epsilon_{0} \frac{d}{d t} \int_{S} \vec{E} \cdot d \vec{a} .
$$

Factorizando obtenemos

$$
\int_{S}\left(\nabla \times \vec{B}-\mu_{0} \vec{J}-\mu_{0} \epsilon_{0} \frac{\partial \vec{E}}{\partial t}\right) \cdot d \vec{a}=0 .
$$

Donde hemos considerado $\frac{d}{d t} \int_{S} \vec{E} \cdot d \vec{a}=\int_{S} \frac{\partial \vec{E}}{\partial t} \cdot d \vec{a}$. La ecuación (25) nos indica que

$$
\nabla \times \vec{B}=\mu_{0} \vec{J}+\mu_{0} \epsilon_{0} \frac{\partial \vec{E}}{\partial t} .
$$

Maestro: Su cálculo hace evidente que el campo magnético es producido también por una variación del campo eléctrico con respecto al tiempo. Me parece que es un buen momento para finalizar la lección. 


\section{Conclusiones}

En este trabajo hemos presentado una revisión didáctica de la ley de Ampère y la ley de Ampère-Maxwell. Consideramos que el diálogo entre maestro y estudiante es un buen recurso didáctico para lograr un aprendizaje significativo de temas de Física.

La ley de Ampère y su generalización con el término de Maxwell son temas de sumo interés en el campo de la ingeniería, no solamente por su evidente utilidad práctica, sino porque nos permiten comprender la importancia de uno de los conceptos fundamentales que es el de campo magnético. Por eso, resulta trascendental cualquier esfuerzo didáctico para abordar tales temas. El presente trabajo pretende coadyuvar en este sentido.

También se hace evidente que el estudiante debe poseer un bagaje de conocimientos previos bien aprendidos antes de emprender la interacción con su interlocutor, de otra manera difícilmente habrá una réplica que le permita reflexionar y comprender los nuevos conceptos que se le intentan enseñar mediante este método, por lo que se deberá recurrir a la lectura y revisión constante de los conceptos necesarios a lo largo del curso, sin obviar ninguno.

Por otra parte, el maestro debe tener una preparación adecuada para propiciar un aprendizaje basado en preguntas (Costenson y Lawson, 1986) y no dejar al estudiante lleno de confusiones y con un entendimiento incompleto de los temas que pretende enseñar. Por esta razón, el dominio de aspectos históricos, conceptuales y matemáticos resultan indispensables para poder planear su clase planteando las preguntas apropiadas que pongan a prueba los conocimientos previos de los estudiantes y/o llevarlos a un conflicto cognitivo que cambie los preconceptos erróneos que posee.

Consideramos que las características de la enseñanza basada en preguntas -donde el maestro guía y conduce al estudiante, mediante el interrogatorio, para obtener su propio conocimientofavorecen la enseñanza de los conceptos físicos, a diferencia del método habitual de enseñanza donde el maestro dicta su lección a manera de conferencia, es decir, mediante un método expositivo, y en el cual la interacción con los alumnos se limita, en el mejor de los casos, a responder las preguntas planteadas por los mismos, sin dar lugar a la reflexión y la retroalimentación (Benítez y Mora, 2010).

Finalmente, es conveniente mencionar que la puesta en práctica de la propuesta didáctica presentada en este trabajo, así como los resultados que se obtengan, serán motivo de un nuevo estudio. Sin embargo, podemos comentar que, al llevarla a la práctica en una clase real, toda propuesta enfrenta imponderables que no se encuentran en el caso ideal, por ejemplo, variables que concurren en el docente como puede ser el conocimiento disciplinar y la experiencia didáctica, factores que condicionan su labor, o los antecedentes académicos del alumnado, además de las dificultades relacionadas con el proceso de formación como la resistencia a los cambios. Si alguno de estos inconvenientes está presente, es claro que, antes de implementar cualquier estrategia didáctica, es necesario resolverlos en la medida de lo posible.

Para la enseñanza de la ley de Ampère y el término de Maxwell basada en preguntas, se propone la siguiente secuencia didáctica (Díaz, 2013):

A. Asegurarse de que el estudiante posee los antecedentes conceptuales necesarios para aprender la ley de Ampère y el término de Maxwell, mediante un examen diagnóstico escrito o, si se prefiere, por medio de un interrogatorio directo en el salón de clases.

B. Desde una perspectiva histórica, abordar los conceptos que se tratarán (la sección 3 de este trabajo es una propuesta a considerar).

C. Preparar las preguntas mediante las cuales guiará al alumno a obtener el conocimiento deseado, considerando que éstas deben permitir al estudiante recordar, detonar un conflicto cognitivo y propiciar la transferencia (las secciones 4 y 5 de este trabajo son propuestas a considerar).

D. Al finalizar el interrogatorio, presentar un resumen de los temas tratados, así como reflexionar acerca de los principales obstáculos enfrentados y de los aciertos durante el desarrollo de esta actividad. 


\section{Referencias}

Arons, A. (1970). Evolución de los conceptos de la física. México: Trillas.

Benítez, Y. y Mora, C. (2010). Enseñanza tradicional vs aprendizaje activo para alumnos de ingeniería. Revista Cubana de Física, 27(2A), 175-179.

Berkson, W. (1985). Las teorías de los campos de fuerza, desde Faraday hasta Einstein. Madrid: Alianza Editorial.

Braun, E. (1992). Electromagnetismo: De la ciencia a la tecnología. México: Fondo de Cultura Económica.

Costenson, K. y Lawson, A.E. (1986). Why isn't inquiry used in more classrooms? The American Biology Teacher, 48(3), 150-158. DOI: 10.2307/4448241

Descartes, R. (2003). Reglas para la dirección del espíritu. Madrid: Alianza Editorial.

Díaz, A. (2013). Secuencias de aprendizaje. ¿Un problema del enfoque de competencias o un reencuentro con perspectivas didácticas? Profesorado, Revista de Currículum y Formación de Profesorado, 17(3), 11-33.

Díaz-Barriga, F. (2010). Estrategias docentes para un aprendizaje significativo. Una interpretación constructivista $\left[3^{\mathrm{a}}\right.$ ed]. México: McGraw Hill.

Glynn, S.M. (1994). Teaching Science With Analogy: A Strategy for Teachers and Textbook Authors. National Reading Research Center Universities of Georgia and Maryland Reading Research Repo, 15, 1-34. Servicio de Reproducción de Documentos ERIC No. ED373306. Recuperado de https://files.eric.ed.gov/fulltext/ED373306.pdf [10 de febrero de 2020].

Holton, G. y Brush, S.G. (1976). Introducción a los conceptos y teorías de las ciencias físicas. Barcelona: Reverté.

Martín, J. y Solbes, J. (2001). Diseño y evaluación de una propuesta para la enseñanza del concepto de campo en Física, Enseñanza de las ciencias, 19(3), 393-403.

Martín del Buey, F. (1997). Transferencia de aprendizaje. En J. Beltrán Llera y J.A. Bueno Álvarez (Eds.), Psicología de la educación (pp. 351-375). México: Alfaomega.

Maxwell, J.C. (1865). A Dynamical Theory of the Electromagnetic Field. Philosophical Transactions of the Royal Society, 155, 459-512. DOI: 10.1098/rstl.1865.0008

Maxwell, J.C. (1954). A Treatise on Electricity and Magnetism. Mineola: Dover Publications.

Moreira, M.A. (2009) Aprendizaje significativo de las ciencias: Condiciones de ocurrencia, progresividad y criticidad. II Jornadas de Enseñanza e Investigación Educativa en el campo de las Ciencias Exactas $y \quad$ Naturales. Recuperado de http://www.memoria.fahce.unlp.edu.ar/trab eventos/ev.612/ev.612.pdf [8 de marzo de 2019].

National Research Council (1996). National Science Education Standards, Washington, DC: National Academy Press.

Pérez, M.C. y Varela, P. (2003). Origen del electromagnetismo. Oersted y Ampère. Madrid: Nivola. Platón. (1984). Diálogos. México: Porrúa.

Polya, G. (1989). Cómo plantear y resolver problemas [15ª ed.]. México: Trillas.

Reitz, J.R, Milford, F.J. y Christy, R.W. (1996). Fundamentos de la teoría electromagnética. España: Addison Wesley Iberoamericana.

Resnick, R., Halliday, D. y Krane, K.S. (2002). Física, volumen 2. México: C.E.C.S.A.

Serway, R.A. (1997). Física, Tomo II. México: McGraw--Hill.

Solbes, J., Pomer, F. y Tarín, F. (1997). Aportaciones de la didáctica de las ciencias y la historia de las ciencias a la enseñanza y el aprendizaje del electromagnetismo. Didáctica de las ciencias experimentales y sociales, 11, 63-75.

Solbes, J. y Martín, J. (1991). Análisis de la introducción del concepto de campo. Revista Española de Física, 5(3), 3439.

Tagüeña, J. y Martina, E. (1988). De la brújula al espín. El magnetismo. México: Fondo de cultura económica. 
Wenning, C.J. (2005a). Levels of inquiry: Hierarchies of pedagogical practices and inquiry processes. Journal of Physics Teacher Education Online, 2(3), 3-11.

Wenning, C.J. (2005b). Whiteboarding and Socratic dialogues: Questions and answers. Journal of Physics Teacher Education Online, 3(1), 3-10.

Wenning, C.J. (2010) Levels of inquiry: Using inquiry spectrum learning sequences to teach science, Journal of Physics Teacher Education Online, 5(3), 11-20.

\section{Cómo CITAR ESTE ARTÍ́CULO}

Sánchez, A, Jaimes, O. y Aguilera, J. R. (2020). La enseñanza basada en preguntas: la ley de Ampère y el término de Maxwell. Didáctica de las ciencias experimentales y sociales, 38, $115-$ 132. DOI: $10.7203 /$ DCES.38.15427. 
